\title{
Morphologic Features Resembling Salivary Gland Adenocarcinoma
}

National Cancer Institute

\section{Source}

National Cancer Institute. Morphologic Features Resembling Salivary Gland

Adenocarcinoma. NCl Thesaurus. Code C161011.

A morphologic finding indicating that an adenocarcinoma which arises from a site other than the salivary glands, resembles a salivary gland adenocarcinoma. 